\title{
Fixed and periodic points of generalized contractions in metric spaces
}

Mujahid Abbas ${ }^{1}$, Basit Ali ${ }^{2}$ and Salvador Romaguera ${ }^{3 *}$

"Correspondence:

sromague@mat.upv.es

${ }^{3}$ Instituto Universitario de

Matemática Pura y Aplicada,

Universitat Politècnica de València, Valencia, 46022, Spain

Full list of author information is

available at the end of the article

\begin{abstract}
Wardowski (Fixed Point Theory Appl. 2012:94, 2012, doi:10.1186/1687-1812-2012-94) introduced a new type of contraction called $F$-contraction and proved a fixed point result in complete metric spaces, which in turn generalizes the Banach contraction principle. The aim of this paper is to introduce $F$-contractions with respect to a self-mapping on a metric space and to obtain common fixed point results. Examples are provided to support results and concepts presented herein. As an application of our results, periodic point results for the $F$-contractions in metric spaces are proved. MSC: $47 \mathrm{H} 10 ; 47 \mathrm{H} 07 ; 54 \mathrm{H} 25$
\end{abstract}

Keywords: F-contraction; property P; property Q; common fixed point

\section{Introduction and preliminaries}

The Banach contraction principle [1] is a popular tool in solving existence problems in many branches of mathematics (see, e.g., [2-4]). Extensions of this principle were obtained either by generalizing the domain of the mapping or by extending the contractive condition on the mappings [5-9]. Initially, existence of fixed points in ordered metric spaces was investigated and applied by Ran and Reurings [10]. Since then, a number of results have been proved in the framework of ordered metric spaces (see [11-18]). Contractive conditions involving a pair of mappings are further additions to the metric fixed point theory and its applications (for details, see [19-23]).

Recently, Wardowski [24] introduced a new contraction called $F$-contraction and proved a fixed point result as a generalization of the Banach contraction principle [1]. In this paper, we introduce an $F$-contraction with respect to a self-mapping on a metric space and obtain common fixed point results in an ordered metric space. In the last section, we give some results on periodic point properties of a mapping and a pair of mappings in a metric space. We begin with some basic known definitions and results which will be used in the sequel. Throughout this article, $\mathbb{N}, \mathbb{R}_{+}, \mathbb{R}$ denote the set of natural numbers, the set of positive real numbers and the set of real numbers, respectively.

Definition 1 Let $f$ and $g$ be self-mappings on a set $X$. If $f x=g x=w$ for some $x$ in $X$, then $x$ is called a coincidence point of $f$ and $g$ and $w$ is called a coincidence point of $f$ and $g$. Furthermore, if $f g x=g f x$ whenever $x$ is a coincidence point of $f$ and $g$, then $f$ and $g$ are called weakly compatible mappings [22].

2013 Abbas et al.: licensee Springer. This is an Open Access article distributed under the terms of the Creative Commons Attribution License (http://creativecommons.org/licenses/by/2.0), which permits unrestricted use, distribution, and reproduction in any medium, provided the original work is properly cited. 
Let $C(f, g)=\{x \in X: f x=g x\}(F(f, g)=\{x \in X: x=f x=g x\})$ denote the set of all coincidence points (the set of all common fixed points) of self-mappings $f$ and $g$.

Definition 2 ([25]) Let $(X, d)$ be a metric space and $f, g: X \rightarrow X$. The mapping $f$ is called a $g$-contraction if there exists $\alpha \in(0,1)$ such that

$$
d(f x, f y) \leq \alpha d(g x, g y)
$$

holds for all $x, y \in X$.

In 1976, Jungck [25] obtained the following useful generalization of the Banach contraction principle.

Theorem 1 Let $g$ be a continuous self-mapping on a complete metric space $(X, d)$. Then $g$ has a fixed point in $X$ if and only if there exists a $g$-contraction mapping $f: X \rightarrow X$ such that $f$ commutes with $g$ and $g(X) \subseteq f(X)$.

Let $\digamma$ be the collection of all mappings $F: \mathbb{R}_{+} \rightarrow \mathbb{R}$ that satisfy the following conditions:

(C1) $F$ is strictly increasing, that is, for all $\alpha, \beta \in \mathbb{R}_{+}$such that $\alpha<\beta$ implies that

$$
F(\alpha)<F(\beta) \text {. }
$$

(C2) For every sequence $\left\{\alpha_{n}\right\}_{n \in \mathbb{N}}$ of positive real numbers, $\lim _{n \rightarrow \infty} \alpha_{n}=0$ and $\lim _{n \rightarrow \infty} F\left(\alpha_{n}\right)=-\infty$ are equivalent.

(C3) There exists $k \in(0,1)$ such that

$$
\lim _{\alpha \rightarrow 0^{+}} \alpha^{k} F(\alpha)=0 .
$$

Definition 3 ([24]) Let $(X, d)$ be a metric space and $F \in F$. A mapping $f: X \rightarrow X$ is said to be an $F$-contraction on $X$ if there exists $\tau>0$ such that

$$
d(f x, f y)>0 \quad \text { implies that } \quad \tau+F(d(f x, f y)) \leq F(d(x, y))
$$

for all $x, y \in X$.

Note that every $F$-contraction is continuous (see [24]). We extend the above definition to two mappings.

Definition 4 Let $(X, d)$ be a metric space, $F \in \digamma$ and $f, g: X \rightarrow X$. The mapping $f$ is said to be an $F$-contraction with respect to $g$ on $X$ if there exists $\tau>0$ such that

$$
\tau+F(d(f x, f y)) \leq F(d(g x, g y))
$$

for all $x, y \in X$ satisfying $\min \{d(f x, f y), d(g x, g y)\}>0$.

By different choices of mappings $F$ in (1) and (2), one obtains a variety of contractions [24]. 
Example 1 Let $F_{1}: \mathbb{R}_{+} \rightarrow \mathbb{R}$ be given by $F_{1}(\alpha)=\ln (\alpha)$. It is clear that $F \in \digamma$. Suppose that $f: X \rightarrow X$ is an $F$-contraction with respect to a self-mapping $g$ on $X$. From (2) we have

$$
\tau+\ln (d(f x, f y)) \leq \ln (d(g x, g y))
$$

which implies that

$$
d(f x, f y) \leq e^{-\tau} d(g x, g y)
$$

Therefore an $F_{1}$-contraction map $f$ with respect to $g$ reduces to a $g$-contraction mapping.

Now we give an example of an $F$-contraction with respect to a self-mapping $g$ on $X$ which is not a $g$-contraction on $X$.

Example 2 Consider the following sequence of partial sums $\left\{S_{n}\right\}_{n \in \mathbb{N}}$ [24, Example 2.5]:

$$
\begin{aligned}
& S_{1}=1, \\
& S_{2}=1+2, \\
& S_{3}=1+2+3, \\
& \cdots \\
& S_{n}=1+2+\cdots+n=\frac{n(n+1)}{2}, \quad n \in \mathbb{N} .
\end{aligned}
$$

Let $X=\left\{S_{n}: n \in \mathbb{N}\right\}$ and $d$ be the usual metric on $X$. Let $f: X \rightarrow X$ and $g: X \rightarrow X$ be defined as

$$
f S_{n}=\left\{\begin{array}{ll}
S_{n-1}, & \text { if } n>1, \\
S_{1}, & \text { if } n=1,
\end{array} \quad g S_{n}= \begin{cases}S_{n+1}, & \text { if } n>1, \\
S_{1}, & \text { if } n=1 .\end{cases}\right.
$$

Let $F_{1}: \mathbb{R}_{+} \rightarrow \mathbb{R}$ be given by $F_{1}(\alpha)=\ln (\alpha)$. As

$$
\lim _{n \rightarrow \infty} \frac{d\left(f S_{n}, f S_{1}\right)}{d\left(g S_{n}, g S_{1}\right)}=\lim _{n \rightarrow \infty} \frac{S_{n-1}-S_{1}}{S_{n+1}-S_{1}}=1,
$$

so $f$ is not a $g$-contraction. If we take $F_{2}(\alpha)=\ln (\alpha)+\alpha$, then $F_{2} \in \digamma$ and $f$ is an $F_{2}$ contraction with respect to a mapping $g$ (taking $\tau=2$ ). Indeed, the following holds:

$$
\frac{d\left(f S_{n}, f S_{1}\right)}{d\left(g S_{n}, g S_{1}\right)} e^{d\left(f S_{n}, f S_{1}\right)-d\left(\left(g S_{n}, g S_{1}\right)\right.}=\frac{S_{n-1}-S_{1}}{S_{n+1}-S_{1}} e^{S_{n-1}-S_{1}-S_{n+1}+S_{1}}=\frac{n^{2}-n-2}{n^{2}+3 n} e^{-4 n-2} \leq e^{-2}
$$

for all $n>1$. For all $m, n \in \mathbb{N}$ with $m>n>1$, we have

$$
\begin{aligned}
& \frac{d\left(f S_{m}, f S_{n}\right)}{d\left(g S_{m}, g S_{n}\right)} e^{d\left(f S_{m}, f S_{n}\right)-d\left(g S_{m}, g S_{n}\right)} \\
& \quad=\frac{S_{m-1}-S_{n-1}}{S_{m+1}-S_{n+1}} e^{S_{m-1}-S_{n-1}-S_{m+1}+S_{n+1}} \\
& =\frac{m^{2}+m-n^{2}-n}{m^{2}+3 m-n^{2}-3 n} e^{-2(m-n)} \leq e^{-2} .
\end{aligned}
$$


Definition 5 ([26], Dominance condition) Let $(X, \preceq)$ be a partially ordered set. A selfmapping $f$ on $X$ is said to be (i) a dominated map if $f x \preceq x$ for each $x$ in $X$, (ii) a dominating map if $x \preceq f x$ for each $x$ in $X$.

Example 3 Let $X=[0,1]$ be endowed with the usual ordering and $f, g: X \rightarrow X$ defined by $g x=x^{n}$ for some $n \in \mathbb{N}$ and $f x=k x$ for some real number $k \geq 1$. Note that

$$
g x=x^{n} \leq x \quad \text { and } \quad x \leq k x=f x
$$

for all $x$ in $X$. Thus $g$ is dominated and $f$ is a dominating map.

Definition 6 Let $(X, \preceq)$ be a partially ordered set. Two mappings $f, g: X \rightarrow X$ are said to be weakly increasing if $f x \preceq g f x$ and $g x \preceq f g x$ for all $x$ in $X$ (see [27]).

Definition 7 Let $X$ be a nonempty set. Then $(X, d, \preceq)$ is called an ordered metric space if $(X, d)$ is a metric space and $(X, \preceq)$ is a partially ordered set.

Definition 8 Let $(X, \preceq)$ be a partial ordered set, then $x, y$ in $X$ are called comparable elements if either $x \preceq y$ or $y \preceq x$ holds true. Moreover, we define $\Delta \subseteq X \times X$ by

$$
\Delta=\{(x, y) \in X \times X: x \preceq y \text { or } y \preceq x\} .
$$

Definition 9 An ordered metric space $(X, d, \preceq)$ is said to have the sequential limit comparison property if for every non-decreasing sequence (non-increasing sequence) $\left\{x_{n}\right\}_{n \in \mathbb{N}}$ in $X$ such that $x_{n} \rightarrow x$ implies that $x_{n} \preceq x\left(x \preceq x_{n}\right)$.

\section{Common fixed point results in ordered metric spaces}

We present the following theorem as a generalization of results in [25] and [24, Theorem 2.1].

Theorem 2 Let $(X, \preceq)$ be a partially ordered set such that there exists a metric $d$ on $X$, and let $f: X \rightarrow X$ be an F-contraction with respect to $g: X \rightarrow X$ on $\Delta$ with $f(X) \subseteq g(X)$. Assume that $f$ is dominating and $g$ is dominated. Then

(a) $f$ and $g$ have a coincidence point in $X$ provided that $g(X)$ is complete and has the sequential limit comparison property.

(b) $C(f, g)$ is well ordered if and only if $C(f, g)$ is a singleton.

(c) $f$ and $g$ have a unique common fixed point if $f$ and $g$ are weakly compatible and $C(f, g)$ is well ordered.

Proof (a) Let $x_{0}$ be an arbitrary point of $X$. Since the range of $g$ contains the range of $f$, there exists a point $x_{1}$ in $X$ such that $f\left(x_{0}\right)=g\left(x_{1}\right)$. As $f$ is dominating and $g$ is dominated, so we have

$$
x_{0} \preceq f x_{0}=g x_{1} \preceq x_{1} .
$$

Hence $\left(x_{0}, x_{1}\right) \in \Delta$. Continuing this process, having chosen $x_{n}$ in $X$, we obtain $x_{n+1}$ in $X$ such that

$$
x_{n} \preceq f x_{n}=g x_{n+1} \preceq x_{n+1} .
$$


So, we obtain $\left(x_{n}, x_{n+1}\right) \in \Delta$ for every $n \in \mathbb{N} \cup\{0\}$. For the sake of simplicity, take

$$
\gamma_{n}=d\left(g x_{n}, g x_{n+1}\right)
$$

for all $n \in \mathbb{N} \cup\{0\}$. If there exists $n_{0} \in \mathbb{N} \cup\{0\}$ for which $x_{n_{0}+1}=x_{n_{0}}$, then $f x_{n_{0}}=g x_{n_{0}+1}$ implies that $f x_{n_{0}+1}=g x_{n_{0}+1}$, that is, $x_{n_{0}+1} \in C(f, g)$. Now we assume that $x_{n+1} \neq x_{n}$ for all $n \in \mathbb{N} \cup\{0\}$. As $f$ is an $F$-contraction with respect to $g$ on $\Delta$, so we obtain

$$
\begin{aligned}
F\left(\gamma_{n}\right) & =F\left(d\left(g x_{n}, g x_{n+1}\right)\right)=F\left(d\left(f x_{n-1}, f x_{n}\right)\right) \\
& \leq F\left(d\left(g x_{n-1}, g x_{n}\right)\right)-\tau \\
& =F\left(d\left(f x_{n-2}, f x_{n-1}\right)\right)-\tau \\
& \leq F\left(d\left(g x_{n-2}, g x_{n-1}\right)\right)-2 \tau \leq \cdots \\
& \leq F\left(d\left(g x_{1}, g x_{2}\right)\right)-(n-1) \tau=F\left(\gamma_{1}\right)-(n-1) \tau .
\end{aligned}
$$

That is,

$$
F\left(\gamma_{n}\right) \leq F\left(\gamma_{1}\right)-(n-1) \tau
$$

On taking limit as $n \rightarrow \infty$, we obtain $\lim _{n \rightarrow \infty} F\left(\gamma_{n}\right)=-\infty$. Hence $\lim _{n \rightarrow \infty} \gamma_{n}=0$ by (C2). Now, by $(\mathrm{C} 3)$, there exists $k \in(0,1)$ such that $\lim _{n \rightarrow \infty} \gamma_{n}^{k} F\left(\gamma_{n}\right)=0$. Note that

$$
\gamma_{n}^{k} F\left(\gamma_{n}\right)-\gamma_{n}^{k} F\left(\gamma_{1}\right) \leq \gamma_{n}^{k}\left(F\left(\gamma_{1}\right)-(n-1) \tau\right)-\gamma_{n}^{k} F\left(\gamma_{1}\right)=-\gamma_{n}^{k}(n-1) \tau \leq 0 .
$$

Taking limit as $n \rightarrow \infty$ in (4), we have $\lim _{n \rightarrow \infty}(n-1) \gamma_{n}^{k}=0$. Consequently, $\lim _{n \rightarrow \infty} n \gamma_{n}^{k}=$ 0 . Thus there exists $n_{1}$ in $\mathbb{N}$ such that $n \gamma_{n}^{k} \leq 1$ for all $n \geq n_{1}$, that is, $\gamma_{n} \leq 1 / n^{1 / k}$ for all $n \geq n_{1}$. Now, for integers $m>n \geq 1$, we obtain

$$
\begin{aligned}
d\left(g x_{n}, g x_{m}\right) & \leq d\left(g x_{n}, g x_{n+1}\right)+d\left(g x_{n+1}, g x_{n+2}\right)+\cdots+d\left(g x_{m-1}, g x_{m}\right) \\
& <\sum_{i=n}^{\infty} \gamma_{i} \leq \sum_{i=n}^{\infty} \frac{1}{i^{\frac{1}{k}}}<\infty .
\end{aligned}
$$

This shows that $\left\{g x_{n}\right\}_{n \in \mathbb{N}}$ is a Cauchy sequence in $g(X)$. As $g(X)$ is complete, so there exists $q$ in $g(X)$ such that $\lim _{n \rightarrow \infty} g x_{n}=q$. Let $p \in X$ be such that $g(p)=q$. The sequential limit comparison property implies that $g x_{n+1} \preceq q$. As $x_{n} \preceq f x_{n}=g x_{n+1} \preceq q=g(p) \preceq p$ so $\left(x_{n}, p\right) \in$ $\Delta$. Hence from (2) we have

$$
F\left(d\left(g x_{n}, f p\right)\right)=F\left(d\left(f x_{n-1}, f p\right)\right) \leq F\left(d\left(g x_{n-1}, g p\right)\right)-\tau .
$$

Since $\lim _{n \rightarrow \infty} d\left(g x_{n-1}, g p\right)=0$, therefore by (C2) we have $\lim _{n \rightarrow \infty} F\left(d\left(g x_{n-1}, g p\right)\right)=-\infty$. Hence $\lim _{n \rightarrow \infty} F\left(d\left(g x_{n}, f p\right)\right)=-\infty$ implies that $\lim _{n \rightarrow \infty} d\left(g x_{n}, f p\right)=0$. That is, $\lim _{n \rightarrow \infty} g x_{n}=$ $f p$. Uniqueness of limit implies $f p=g p$, that is, $p \in C(f, g)$.

(b) Now suppose that $C(f, g)$ is well ordered. We prove that $C(f, g)$ is a singleton. Assume on the contrary that there exists another point $w$ in $X$ such that $f w=g w$ with $w \neq p$. Since $C(f, g)$ is well ordered, so $(w, p) \in \Delta$. Now from (2) we have

$$
\tau \leq F(d(g w, g p))-F(d(f w, f p))=0,
$$


a contradiction. Therefore $w=p$. Hence $f$ and $g$ have a unique coincidence point $p$ in $X$. The converse follows immediately.

(c) Now if $f$ and $g$ are weakly compatible mappings, then we have $f q=f g p=g f p=g q$, that is, $q$ is the coincidence point of $f$ and $g$. But $q$ is the only point of coincidence of $f$ and $g$, so $f q=g q=q$. Hence $q$ is the unique common fixed point of $f$ and $g$.

Example 4 Let $X=[0,5]$ be endowed with usual metric and usual order. Define mappings $f, g: X \rightarrow X$ by

$$
g x=\left\{\begin{array}{ll}
0 & \text { if } x \in[0,3), \\
3 & \text { if } x \in[3,5), \\
5 & \text { if } x=5,
\end{array} \quad f x= \begin{cases}3 & \text { if } x \in[0,3) \\
5 & \text { if } x \in[3,5]\end{cases}\right.
$$

Clearly, $g$ is dominated and $f$ is dominating. Define $F: \mathbb{R}_{+} \rightarrow \mathbb{R}$ as $F(x)=\ln (x)$. If $x \in$ $[0,3)$ and $y \in[3,5)$, then

$$
\begin{aligned}
F(d(f x, f y)) & =F(d(3,5))=F(2)=\ln (2) \approx 0.693 \\
& <F(d(g x, g y))=F(d(0,3)) \\
& =F(3)=\ln (3) \approx 1.098 .
\end{aligned}
$$

Hence, for $\tau \in(0,0.40]$, inequality (2) is satisfied. Similarly, for $x \in[0,3)$ and $y=5$, we have

$$
\begin{aligned}
F(d(f x, f y)) & =F(d(3,5))=F(2)=\ln (2) \approx 0.693 \\
& <F(d(g x, g y))=F(d(0,5)) \\
& =F(5)=\ln (5) \approx 1.6094 .
\end{aligned}
$$

Hence, for $\tau \in(0,0.9164]$, inequality (2) is satisfied. We can take a $\tau \in(0,0.40]$ so that

$$
\tau+F(d(f x, f y)) \leq F(d(g x, g y))
$$

is satisfied for all $x, y \in[0,5]$, whenever $\min \{d(f x, f y), d(g x, g y)\}>0$. Hence $f$ is an $F$ contraction with respect to $g$ on $[0,5]$. Hence all the conditions of Theorem 2 are satisfied. Moreover, $x=5$ is the coincidence point of $f$ and $g$. Also note that $f$ and $g$ are weakly compatible and $x=5$ is the common fixed point of $g$ and $f$ as well.

Now we give a common fixed point result without imposing any type of commutativity condition for self-mappings $f$ and $g$ on $X$. Moreover, we relax the dominance conditions on $f$ and $g$ as well.

Theorem 3 Let $(X, \preceq)$ be a partially ordered set such that there exists a complete metric d on $X$. If self-mappings $f$ and $g$ on $X$ are weakly increasing and for some $\tau>0$ satisfy

$$
\tau+F(d(f x, g y)) \leq F(d(x, y))
$$


for all $(x, y) \in \Delta$ such that $\min \{d(f x, g y), d(x, y)\}>0$, then $F(f, g) \neq \emptyset$, provided that $X$ has the sequential limit comparison property. Further, $f$ and $g$ have a unique common fixed point if and only if $F(f, g)$ is well ordered.

Proof Let $x_{0}$ be an arbitrary point of $X$. Define a sequence $\left\{x_{n}\right\}_{n \in \mathbb{N}}$ in $X$ as follows: $x_{2 n+1}=$ $f x_{2 n}$ and $x_{2 n+2}=g x_{2 n+1}$. Since $f$ and $g$ are weakly increasing, we have $x_{2 n+1}=f x_{2 n} \preceq g f x_{2 n}=$ $g x_{2 n+1}=x_{2 n+2}$ and $x_{2 n+2}=g x_{2 n+1} \preceq f g x_{2 n+1}=f x_{2 n+2}=x_{2 n+3}$. Hence $\left(x_{2 n+1}, x_{2 n+2}\right) \in \Delta$ and $\left(x_{2 n+2}, x_{2 n+3}\right) \in \Delta$ for every $n \in \mathbb{N} \cup\{0\}$. Now define

$$
\gamma_{2 n}=d\left(x_{2 n+1}, x_{2 n+2}\right)
$$

for all $n \in \mathbb{N} \cup\{0\}$. Using (5) the following holds for every $n \in \mathbb{N} \cup\{0\}$ :

$$
\begin{gathered}
F\left(\gamma_{2 n}\right)=F\left(d\left(x_{2 n+1}, x_{2 n+2}\right)\right)=F\left(d\left(f x_{2 n}, g x_{2 n+1}\right)\right) \\
\leq F\left(d\left(x_{2 n}, x_{2 n+1}\right)\right)-\tau=F\left(\gamma_{2 n-1}\right)-\tau .
\end{gathered}
$$

Similarly,

$$
\begin{aligned}
F\left(\gamma_{2 n+1}\right) & =F\left(d\left(x_{2 n+3}, x_{2 n+2}\right)\right)=F\left(d\left(f x_{2 n+2}, g x_{2 n+1}\right)\right) \\
& \leq F\left(d\left(x_{2 n+1}, x_{2 n+2}\right)\right)-\tau=F\left(\gamma_{2 n}\right)-\tau .
\end{aligned}
$$

Therefore, for all $n \in \mathbb{N} \cup\{0\}$, we have

$$
\begin{aligned}
F\left(\gamma_{n}\right) & \leq F\left(\gamma_{n-1}\right)-\tau \leq F\left(\gamma_{n-2}\right)-2 \tau \leq \cdots \\
& \leq F\left(d\left(x_{1}, x_{2}\right)\right)-n \tau=F\left(\gamma_{0}\right)-n \tau .
\end{aligned}
$$

Thus

$$
F\left(\gamma_{n}\right) \leq F\left(\gamma_{0}\right)-n \tau
$$

Taking limit as $n \rightarrow \infty$ in (7), we get

$$
\lim _{n \rightarrow \infty} F\left(\gamma_{n}\right)=-\infty
$$

By (C2) and (C3) we get $\lim _{n \rightarrow \infty} \gamma_{n}=0$ and $k \in(0,1)$ such that $\lim _{n \rightarrow \infty} \gamma_{n}^{k} F\left(\gamma_{n}\right)=0$. Note that

$$
\gamma_{n}^{k} F\left(\gamma_{n}\right)-\gamma_{n}^{k} F\left(\gamma_{0}\right) \leq \gamma_{n}^{k}\left(F\left(\gamma_{0}\right)-n \tau\right)-\gamma_{n}^{k} F\left(\gamma_{0}\right)=-\gamma_{n}^{k} n \tau \leq 0 .
$$

By taking limit as $n \rightarrow \infty$ in (8), we get $\lim _{n \rightarrow \infty} n \gamma_{n}^{k}=0$. This implies that there exists $n_{1}$ such that $n \gamma_{n}^{k} \leq 1$ for all $n \geq n_{1}$. Consequently, we obtain $\gamma_{n} \leq 1 / n^{1 / k}$ for all $n \geq n_{1}$. Now, for integers $m>n \geq 1$, we have

$$
d\left(x_{n}, x_{m}\right) \leq d\left(x_{n}, x_{n+1}\right)+d\left(x_{n+1}, x_{n+2}\right)+\cdots+d\left(x_{m-1}, x_{m}\right)<\sum_{i=n}^{\infty} \gamma_{i} \leq \sum_{i=n}^{\infty} \frac{1}{i \frac{1}{k}}<\infty
$$


This shows that $\left\{x_{n}\right\}_{n \in \mathbb{N}}$ is a Cauchy sequence in $X$, so there exists $p$ in $X$ such that $\lim _{n \rightarrow \infty} x_{n}=p$. As $X$ has the sequential limit comparison property, so $\left(x_{n}, p\right),\left(x_{2 n}, p\right)$, $\left(x_{2 n+1}, p\right) \in \Delta$. Therefore

$$
\lim _{n \rightarrow \infty} F\left(d\left(x_{2 n+1}, g p\right)\right)=\lim _{n \rightarrow \infty} F\left(d\left(f x_{2 n}, g p\right)\right) \leq F\left(d\left(x_{2 n}, p\right)\right)-\tau .
$$

Since $\lim _{n \rightarrow \infty} d\left(x_{2 n}, p\right)=0$, by (C2) we have $\lim _{n \rightarrow \infty} F\left(d\left(x_{2 n}, p\right)\right)=-\infty$. This implies $\lim _{n \rightarrow \infty} F\left(d\left(x_{2 n+1}, g p\right)\right)=-\infty$, which further implies that $\lim _{n \rightarrow \infty} d\left(x_{2 n+1}, g p\right)=0$. Hence $d(p, g p)=0$ and $p=g p$. Similarly, we obtain $p=f p$. This shows that $p$ is a common fixed point of $g$ and $f$. Now suppose that $F(f, g)$ is well ordered. We prove that $F(f, g)$ is a singleton. Assume on the contrary that there exists another point $q$ in $X$ such that $q=f q=g q$ with $q \neq p$. Obviously, $(q, p) \in \Delta$. So, from (5) we have $\tau \leq F(d(q, p))-F(d(f q, g p))=0$, a contradiction. Therefore $q=p$. Hence $g$ and $f$ have a unique common fixed point $p$ in $X$. The converse follows immediately.

\section{Periodic point results in metric spaces}

If $x$ is a fixed point of the self-mapping $f$, then $x$ is a fixed point of $f^{n}$ for every $n \in \mathbb{N}$, but the converse is not true. In the sequel, we denote by $F(f)$ the set of all fixed points of $f$.

Example 5 Let $f:[0,1] \rightarrow[0,1]$ be given by

$$
f(x)=1-x
$$

Then $f$ has a unique fixed point $x=1 / 2$. Note that $f^{n} x=x$ holds for every even natural number $n$ and $x$ in $[0,1]$. On the other hand, define a mapping $g:[0, \pi] \rightarrow[0, \pi]$ as

$$
g(x)=\cos x .
$$

Then $g$ has the same fixed point as $g^{n}$ for every $n$.

Definition 10 The self-mapping $f$ is said to have the property $P$ if $F\left(f^{n}\right)=F(f)$ for every $n \in \mathbb{N}$. A pair $(f, g)$ of self-mappings is said to have the property $Q$ if $F(f) \cap F(g)=F\left(f^{n}\right) \cap$ $F\left(g^{n}\right)$.

For further details on these properties, we refer to [20,28].

Let $(X, d)$ be a metric space and $f: X \rightarrow X$ be a self-mapping. The set $O(x)=\{x, f x, \ldots$, $\left.f^{n} x, \ldots\right\}$ is called the orbit of $x$ [29]. A mapping $f$ is called orbitally continuous at $p$ if $\lim _{n \rightarrow \infty} f^{n} x=p$ implies that $\lim _{n \rightarrow \infty} f^{n+1} x=f p$. A mapping $f$ is orbitally continuous on $X$ if $f$ is orbitally continuous for all $x \in X$.

In this section we prove some periodic point results for self-mappings on complete metric spaces.

Theorem 4 Let $X$ be a nonempty set such that there exists a complete metric $d$ on $X$. Suppose that $f: X \rightarrow X$ satisfies

$$
\tau+F\left(d\left(f x, f^{2} x\right)\right) \leq F(d(x, f x))
$$


for some $\tau>0$ and for all $x$ in $X$ such that $d\left(f x, f^{2} x\right)>0$. Then $f$ has the property $P$ provided that $f$ is orbitally continuous on $X$.

Proof First we show that $F(f) \neq \emptyset$. Let $x_{0} \in X$. Define a sequence $\left\{x_{n}\right\}_{n \in \mathbb{N}}$ in $X$, such that $x_{n+1}=f x_{n}$, for all $n \in \mathbb{N} \cup\{0\}$. Denote $\gamma_{n}=d\left(x_{n}, x_{n+1}\right)$ for all $n \in \mathbb{N} \cup\{0\}$. If there exists $n_{0} \in \mathbb{N} \cup\{0\}$ for which $x_{n_{0}+1}=x_{n_{0}}$, then $f x_{n_{0}}=x_{n_{0}}$ and the proof is finished. Suppose that $x_{n+1} \neq x_{n}$ for all $n \in \mathbb{N} \cup\{0\}$. Using (9), we obtain

$$
\begin{aligned}
F\left(\gamma_{n}\right) & =F\left(d\left(x_{n}, x_{n+1}\right)\right)=F\left(d\left(f x_{n-1}, f^{2} x_{n-1}\right)\right) \\
& \leq F\left(d\left(x_{n-1}, f x_{n-1}\right)\right)-\tau=F\left(d\left(f x_{n-2}, f^{2} x_{n-2}\right)\right)-\tau \\
& \leq F\left(d\left(x_{n-2}, f x_{n-2}\right)\right)-2 \tau \leq \cdots \\
& \leq F\left(d\left(x_{1}, x_{2}\right)\right)-(n-1) \tau \\
& =F\left(d\left(x_{0}, f^{2} x_{1}\right)\right)-(n-1) \tau \leq F\left(d\left(x_{0}, x_{1}\right)\right)-n \tau \\
& =F\left(\gamma_{0}\right)-n \tau
\end{aligned}
$$

for every $n \in \mathbb{N} \cup\{0\}$. By taking limit as $n \rightarrow \infty$ in the above inequality, we obtain that $\lim _{n \rightarrow \infty} F\left(\gamma_{n}\right)=-\infty$, which together with (C2) gives $\lim _{n \rightarrow \infty} \gamma_{n}=0$. From (C3), there exists $k \in(0,1)$ such that $\lim _{n \rightarrow \infty} \gamma_{n}^{k} F\left(\gamma_{n}\right)=0$. Note that

$$
\begin{aligned}
\gamma_{n}^{k} F\left(\gamma_{n}\right)-\gamma_{n}^{k} F\left(\gamma_{0}\right) & \leq \gamma_{n}^{k}\left(F\left(\gamma_{0}\right)-n \tau\right)-\gamma_{n}^{k} F\left(\gamma_{0}\right) \\
& =-\gamma_{n}^{k} n \tau \leq 0 .
\end{aligned}
$$

On taking limit as $n \rightarrow \infty$, we get $\lim _{n \rightarrow \infty} n \gamma_{n}^{k}=0$. Hence there exists $n_{1}$ such that $n \gamma_{n}^{k} \leq 1$ for all $n \geq n_{1}$. Consequently $\gamma_{n} \leq 1 / n^{1 / k}$ for all $n \geq n_{1}$. Now, for integers $m>n \geq 1$ such that

$$
\begin{aligned}
d\left(f^{n} x_{0}, f^{m} x_{0}\right) & =d\left(x_{n}, x_{m}\right) \leq d\left(x_{n}, x_{n+1}\right)+d\left(x_{n+1}, x_{n+2}\right)+\cdots+d\left(x_{m-1}, x_{m}\right) \\
& <\sum_{i=n}^{\infty} \gamma_{i} \leq \sum_{i=n}^{\infty} \frac{1}{i \frac{1}{k}}<\infty .
\end{aligned}
$$

This shows that $\left\{f^{n} x_{0}\right\}_{n \in \mathbb{N}}$ is a Cauchy sequence. Since $\left\{f^{n} x_{0}: n \in \mathbb{N}\right\} \subseteq O\left(x_{0}\right) \subseteq X$ and $X$ is complete, which implies that there exists $x$ in $X$ such that $\lim _{n \rightarrow \infty} f^{n} x_{0}=x$. Since $f$ is orbitally continuous at $x$, so $x=\lim _{n \rightarrow \infty} f^{n} x_{0}=f\left(\lim _{n \rightarrow \infty} f^{n-1} x_{0}\right)=f x$. Hence $f$ has a fixed point and $F\left(f^{n}\right)=F(f)$ is true for $n=1$. Now assume $n>1$. Suppose on the contrary that $u \in F\left(f^{n}\right)$ but $u \notin F(f)$, then $d(u, f u)=\alpha>0$. Now consider

$$
\begin{aligned}
F(\alpha) & =F(d(u, f u))=F\left(d\left(f\left(f^{n-1} u\right), f^{2}\left(f^{n-1} u\right)\right)\right) \\
& \leq F\left(d\left(f^{n-1} u, f^{n} u\right)\right)-\tau \\
& \leq F\left(d\left(f^{n-2} u, f^{n-1} u\right)\right)-2 \tau \leq \cdots \\
& \leq F(d(u, f u))-n \tau .
\end{aligned}
$$

Thus $F(\alpha) \leq \lim _{n \rightarrow \infty} F(d(u, f u))-n \tau=-\infty$. Hence $F(\alpha)=-\infty$. By $(C 2) \alpha=0$, a contradiction. So $u \in F(f)$. 
Theorem 5 Let $(X, \preceq)$ be a partially ordered set such that there exists a complete metric $d$ on $X$ and $f, g$ self-mappings on $X$. Further assume that $f, g$ are weakly increasing and satisfy

$$
\tau+F(d(f x, g y)) \leq F(d(x, y))
$$

for some $\tau>0$, for all $x, y$ in $X$ such that $\min \{d(f x, g y), d(x, y)\}>0$. Then $f$ and $g$ have the property $Q$ provided that $X$ has the sequential limit comparison property.

Proof By Theorem 3, $f$ and $g$ have a common fixed point. Suppose on the contrary that

$$
u \in F\left(f^{n}\right) \cap F\left(g^{n}\right)
$$

but $u \notin F(f) \cap F(g)$, then there are three possibilities (a) $u \in F(f) \backslash F(g)$, (b) $u \in F(g) \backslash F(f)$, (c) $u \notin F(f)$ and $u \notin F(g)$. Without loss of generality, let $u \notin F(g)$, that is, $d(u, g u)=\alpha>0$, so we get

$$
\begin{aligned}
F(\alpha) & =F(d(u, g u))=F\left(d\left(f\left(f^{n-1} u\right), g\left(g^{n} u\right)\right)\right) \\
& \leq F\left(d\left(f^{n-1} u, g^{n} u\right)\right)-\tau \\
& \leq F\left(d\left(f^{n-2} u, g^{n-1} u\right)\right)-2 \tau \leq \cdots \\
& \leq F(d(u, g u))-n \tau .
\end{aligned}
$$

As $\lim _{n \rightarrow \infty} F(d(u, g u))-n \tau=-\infty$, so we have $F(\alpha)=-\infty$. By $(\mathrm{C} 2) \alpha=0$, a contradiction. Hence $u \in F(g) \cap F(f)$.

\section{Competing interests}

The authors declare that they have no competing interests.

Authors' contributions

The three authors contributed equally in writing this article. They read and approved the final manuscript.

\section{Author details}

${ }^{1}$ Department of Mathematics and Applied Mathematics, University of Pretoria, Hatfield, Pretoria, South Africa.

${ }^{2}$ Department of Mathematics, Syed Babar Ali School of Science and Engineering, Lahore University of Management Sciences, Lahore, 54792, Pakistan. ${ }^{3}$ Instituto Universitario de Matemática Pura y Aplicada, Universitat Politècnica de València, Valencia, 46022, Spain.

\section{Acknowledgements}

The third author thanks for the support of the Ministry of Economy and Competitiveness of Spain, Grant MTM2012-37894-C02-01.

Received: 1 July 2013 Accepted: 8 October 2013 Published: 07 Nov 2013

\section{References}

1. Banach, S: Sur les opérations dans les ensembles abstraits et leur applications aux équations intégrales. Fundam. Math. 3, 133-181 (1922)

2. Dix, JG, Karakostas, GL: A fixed point theorem for S-type operators on Banach spaces and its applications to boundary-value problems. Nonlinear Anal. 71, 3872-3880 (2009)

3. Latrach, K, Taoudib, MA, Zeghalc, A: Some fixed point theorems of the Schauder and the Krasnosel'skii type and application to nonlinear transport equations. J. Differ. Equ. 221, 256-271 (2006)

4. Rousseau, C: Point fixe de Banach (in French). Accromath 5, hiver-printemps (2010) (www.accromath.ca)

5. Arandjelović, I, Kadelburg, Z, Radenović, S: Boyd-Wong-type common fixed point results in cone metric spaces. Appl. Math. Comput. 217, 7167-7171 (2011)

6. Boyd, DW, Wong, JSW: On nonlinear contractions. Proc. Am. Math. Soc. 20, 458-464 (1969) 
7. Huang, LG, Zhang, X: Cone metric spaces and fixed point theorems of contractive mappings. J. Math. Anal. Appl. 332 1468-1476 (2007)

8. Rakotch, E: A note on contractive mappings. Proc. Am. Math. Soc. 13, 459-465 (1962)

9. Tarafdar, E: An approach to fixed-point theorems on uniform spaces. Trans. Am. Math. Soc. 191, $209-225$ (1974)

10. Ran, ACM, Reurings, MCB: A fixed point theorem in partially ordered sets and some application to matrix equations. Proc. Am. Math. Soc. 132, 1435-1443 (2004)

11. Abbas, M, Khamsi, MA, Khan, AR: Common fixed point and invariant approximation in hyperbolic ordered metric spaces. Fixed Point Theory Appl. 2011, Article ID 25 (2011). doi:10.1186/1687-1812-2011-25

12. Altun, I, Simsek, H: Some fixed point theorems on ordered metric spaces and application. Fixed Point Theory Appl. 2010, Article ID $621492(2010)$

13. Amini-Harandi, A, Emami, H: A fixed point theorem for contraction type maps in partially ordered metric spaces and application to ordinary differential equations. Nonlinear Anal. 72, 2238-2242 (2010)

14. Ćirić, L, Abbas, M, Saadati, R, Hussain, N: Common fixed points of almost generalized contractive mappings in ordered metric spaces. Appl. Math. Comput. 217, 5784-5789 (2011)

15. Harjani, J, Sadarangani, K: Fixed point theorems for weakly contractive mappings in partially ordered sets. Nonlinear Anal. 71, 3403-3410 (2009)

16. Kadelburg, Z, Pavlović, M, Radenović, S: Common fixed point theorems for ordered contractions and quasicontractions in ordered cone metric spaces. Comput. Math. Appl. 59, 3148-3159 (2010)

17. Nieto, JJ, Rodríguez-López, R: Contractive mapping theorems in partially ordered sets and applications to ordinary differential equations. Order 22, 223-239 (2005)

18. Samet, B: Coupled fixed point theorems for a generalized Meir-Keeler contraction in partially ordered metric spaces. Nonlinear Anal. 72, 4508-4517 (2010)

19. Al-Thagafi, MA: Common fixed points and best approximation. J. Approx. Theory 85, 318-320 (1996)

20. Beg, I, Abbas, M: Coincidence point and invariant approximation for mappings satisfying generalized weak contractive condition. Fixed Point Theory Appl. 2006, Article ID 74503 (2006)

21. Jungck, G: Compatible mappings and common fixed points. Int. J. Math. Math. Sci. 9, 771-779 (1986)

22. Jungck, G: Common fixed points for noncontinuous nonself maps on nonmetric spaces. Far East J. Math. Sci. 4, 199-215 (1996)

23. Sessa, S: On a weak commutativity condition of mappings in fixed point consideration. Publ. Inst. Math. (Belgr.) 32, 149-153 (1982)

24. Wardowski, D: Fixed points of new type of contractive mappings in complete metric spaces. Fixed Point Theory Appl. (2012). doi:10.1186/1687-1812-2012-94

25. Jungck, G: Commuting mappings and fixed points. Am. Math. Mon. 83, 261-263 (1976)

26. Abbas, M, Nazir, T, Radenović, S: Common fixed points of four maps in partially ordered metric spaces. Appl. Math. Lett. 24, 1520-1526 (2011)

27. Altun, I, Damjanović, B, Djorić, D: Fixed point and common fixed point theorems on ordered cone metric spaces. Appl. Math. Lett. 23, 310-316 (2010)

28. Jeong, GS, Rhoades, BE: Maps for which $F(T)=F\left(T^{n}\right)$. In: Fixed Point Theory and Applications, vol. 6, pp. 71-105. Nova Science Publishers, New York (2007)

29. Hicks, TL, Rhoades, BE: A Banach type fixed point theorem. Math. Jpn. 24(3), 327-330 (1979)

10.1186/1687-1812-2013-243

Cite this article as: Abbas et al.: Fixed and periodic points of generalized contractions in metric spaces. Fixed Point

Theory and Applications 2013, 2013:243

\section{Submit your manuscript to a SpringerOpen ${ }^{\circ}$ journal and benefit from:}

- Convenient online submission

- Rigorous peer review

- Immediate publication on acceptance

- Open access: articles freely available online

- High visibility within the field

- Retaining the copyright to your article

Submit your next manuscript at $>$ springeropen.com 